\title{
A FUNCTION IS PERRON INTEGRABLE IF IT HAS LOCALLY SMALL RIEMANN SUMS
}

\author{
ARLO W. SCHURLE
}

(Received 5 June 1984)

Communicated by J. H. Chabrowski

\begin{abstract}
We say that a function has locally small Riemann sums on an interval if for each point $x$ in the interval, and for each positive number $\varepsilon$, all sufficiently fine partitions of intervals lying in a neighborhood of $x$ but not containing $x$ have Riemann sums of absolute value less than $\varepsilon$. The main result is then as the title states. We use the generalized Riemann approach to Perron integration, assuming that functions are measurable only to insure that conditions involving the positive and negative parts of the functions are satisfied.
\end{abstract}

1980 Mathematics subject classification (Amer. Math. Soc.): 26 A 39, 26 A 42.

\section{Introduction and background}

This paper uses the generalized Riemann approach to the Perron-Stieltjes and Lebesgue-Stieltjes integrals. This approach originated with ideas of Henstock, Kurzweil and McShane and has been presented by McLeod in [1] and by Pfeffer in [3], to which the reader is referred for more details than we provide here.

This approach to integration and the specific problems which motivated this paper were brought to the author's attention in a seminar at the University of Petroleum and Minerals conducted by W. Pfeffer, to whom the author is grateful for several enlightening conversations.

Our interest is in Perron-Stieltjes and Lebesgue-Stieltjes integrals on real intervals, so by an interval $J$ we will always mean a nondegenerate closed

(C) 1986 Australian Mathematical Society $0263-6115 / 86 \$ A 2.00+0.00$ 
bounded interval of real numbers, and $J^{0}$ will denote its interior. Further, all functions will be real-valued.

By a subpartition $P$ of an interval $J$ we mean a set $\left\{I_{1}, I_{2}, \ldots, I_{n}\right.$; $\left.x_{1}, x_{2}, \ldots, x_{n}\right\}$, where $I_{1}, I_{2}, \ldots, I_{n}$ are nonoverlapping intervals (their interiors are pairwise disjoint), and where $x_{1}, x_{2}, \ldots, x_{n}$ are points of the interval $J$. We say that $x_{1}, x_{2}, \ldots, x_{n}$ are the points of $P$ and that $x_{i}$ is the point of $P$ corresponding to $I_{i}$. If each point $x_{i}$ belongs to its corresponding interval $I_{i}$, then we say that $P$ is a Perron subpartition. If $\delta$ is a positive function on the interval $J$ then we say that $P$ is $\delta$-fine if $I_{i}$ is contained in $\left(x_{i}-\delta\left(x_{i}\right), x_{i}+\delta\left(x_{i}\right)\right)$ for each $i$. Finally, a partition of an interval $J$ is a subpartition whose intervals have union $J$.

We use $\alpha$ to denote a nondecreasing function on the real numbers which we assume to be fixed throughout this paper. Then we set $\alpha([a, b])=\alpha(b)-\alpha(a)$. As usual this induces a Borel measure on the interval, so we may speak of a function being $\alpha$-measurable.

Let $f$ be any function on an interval $J$ and let $P$ be a partition of $J$, say $P=\left\{I_{1}, I_{2}, \ldots, I_{n} ; x_{1}, x_{2}, \ldots, x_{n}\right\}$. We denote $\sum_{i=1}^{n} f\left(x_{i}\right) \alpha\left(I_{i}\right)$ by $\sigma(f, P, \alpha)$, or just $\sigma(f, P)$ if no confusion will result. If there is a number $A$ such that for each $\varepsilon>0$ there is a positive function $\delta$ on $J$ for which $|\sigma(f, P)-A|<\varepsilon$ whenever $P$ is a $\delta$-fine partition of $J$, then we say that $f$ is Lebesgue-Stieltjes integrable with respect to $\alpha$ (or just $L$-integrable) on the interval $J$. If we require only that $\sigma(f, P)$ is near $A$ for $P$ a $\delta$-fine Perron partition of $J$, then we say that $f$ is Perron-Stieltjes integrable with respect to $\alpha$ (or just $P$-integrable) on $J$. We then denote $A$ by $L \int f d \alpha$ in the Lebesgue case and by $P \int f d \alpha$ in the Perron case.

Again let $f$ be any function on an interval. We use $F^{+}$and $F^{-}$to denote the sets $\{x \mid f(x) \geqslant 0\}$ and $\{x \mid f(x) \leqslant 0\}$, respectively. Analogously we use $f^{+}$for the function $(f+|f|) / 2$ and $f^{-}$for the function $(|f|-f) / 2$. Finally, we use $\operatorname{supp}(f)$ to denote the closure of the set of points at which $f$ has non-zero value.

We assume throughout that functions whose integrability with respect to $\alpha$ is in question are $\alpha$-measurable. We do not use this assumption directly, but rather use it to enable us to assume first, that $F^{+}$and $F^{-}$are $G_{\delta^{-}}$sets and second, that if $f^{+}$ is not integrable, then for any positive function $\delta$ and any number $M$ there is a $\delta$-fine partition $P$ for which $\sigma\left(f^{+}, P\right)>M$. The first assertion is a standard measure-theoretic result and the second follows from the fact that the classical and generalized Riemann approaches to integration agree. (See [5].)

We can now make precise the property referred to in the title. We say that a function $f$ has locally small Riemann sums on an interval $I$ with respect to $\alpha$ if for each $b$ in $I$ and each positive $\varepsilon$ there is a positive function $\delta$ on $I$ for which $|\sigma(f, P)|<\varepsilon$ whenever $P$ is a $\delta$-fine Perron partition of an interval lying in $(b-\delta(b), b+\delta(b))-\{b\}$. We note that the function $\delta$ may depend on both $b$ and $\varepsilon$. 
We can now state our main result.

Main Theorem. An $\alpha$-measurable function $f$ on an interval I is Perron-Stieltjes integrable on I with respect to $\alpha$ if and only if $f$ has locally small Riemann sums with respect to $\alpha$ on $I$.

We break the proof into several sections, some of which contain results that may be of independent interest.

\section{Proof of necessity}

Let $b$ be a point of the interval $I=[a, c]$ and let $\varepsilon$ be positive. Since $f$ is $P$-integrable with respect to $\alpha$, there is a positive function $\delta$ on $I$ such that if $P$ and $P^{\prime}$ are $\delta$-fine Perron partitions of $I$, then $\left|\sigma(f, P)-\sigma\left(f, P^{\prime}\right)\right|<\varepsilon / 2$. We may assume that $\delta(b)$ is so small that $|f(b)| \alpha([b-\delta(b), s])<\varepsilon / 2$ whenever $b-\delta(b)<s<b$, and that $|f(b)| \alpha([t, b+\delta(b)])<\varepsilon / 2$ whenever $b<t<b+$ $\delta(b)$.

Let $Q$ be a $\delta$-fine partition of an interval $[r, s]$ in $[b-\delta(b), b)$. Use the interval $[s, b]$ with corresponding point $b, a \delta$-fine partition of $[a, r]$, and a $\delta$-fine partition of $[b, c]$, together with $Q$, to obtain a $\delta$-fine partition $P$ of $I$.

Use the same partitions of $[a, r]$ and $[b, c]$ and the interval $[r, b]$ with corresponding point $b$ to obtain a $\delta$-fine partition $P^{\prime}$ of $I$. Now $\mid \sigma(f, P)-$ $\sigma\left(f, P^{\prime}\right) \mid<\varepsilon / 2$ and $\left|\sigma(f, P)-\sigma\left(f, P^{\prime}\right)\right|=\mid \sigma(f, Q)+f(b) \alpha([s, b])-$ $f(b) \alpha([r, b])|=| \sigma(f, Q)-f(b) \alpha([r, s]) \mid$. Since $|f(b) \alpha([r, s])|<\varepsilon / 2$, we obtain $|\sigma(f, Q)|<\varepsilon$. We treat intervals in $(b, b+\delta(b))$ in exactly the same way, and so our result follows.

\section{Local Lebesgue integrability of $f$}

In this section we prove that if $f$ has locally small Riemann sums, then the set of points of $\operatorname{supp}(f)$ which have neighborhoods on which $f$ is Lebesgue integrable with respect to $\alpha$ is dense in $\operatorname{supp}(f)$. Note the similarity of this result with the main result of [5].

Let $b$ be a point of $\operatorname{supp}(f)$. If $b$ is an isolated point of $\operatorname{supp}(f)$, then certainly $f$ is Lebesgue integrable on a neighborhood of $b$. So suppose that $b$ is a limit point of $\operatorname{supp}(f)$ and let $U$ be a neighborhood of $b$.

Since $f$ has locally small Riemann sums, there is a positive function $\delta$ on $I$ such that $|\sigma(f, P)|<1$ whenever $P$ is a $\delta$-fine Perron partition of an interval 
contained in $(b-\delta(b), b+\delta(b))-\{b\}$. We may certainly assume that $(b-\delta(b), b+\delta(b))$ is contained in $U$ and that $(b-\delta(b), b)$ contains points of $\operatorname{supp}(f)$.

Suppose that $f$ is Lebesgue integrable on no neighborhood of any point of $\operatorname{supp}(f)$ in $(b-\delta(b), b)$. Let $J$ be an interval which is a neighborhood of such a point and which lies in $(b-\delta(b), b)$. Either $f^{+}$or $f^{-}$is not Lebesgue integrable on $J$. If $f^{-}$is not but $f^{+}$is, then for some positive function $\delta^{\prime}<\delta$, and for some number $M$, we must have $\sigma\left(f^{+}, P\right)<M$ whenever $P$ is a $\delta^{\prime}$-fine Perron partition of $J$. But there is a $\delta^{\prime}$-fine partition $P$ of $J$ for which $\sigma\left(f^{-}, P\right)>M+10$. Then $|\sigma(f, P)|=\left|\sigma\left(f^{+}, P\right)-\sigma\left(f^{-}, P\right)\right|>10$, contradicting the choice of $\delta$. Similarly $f^{+}$cannot fail to be Lebesgue integrable while $f^{-}$is. We conclude from this that neither $f^{+}$nor $f^{-}$is integrable on any neighborhood of a point of $\operatorname{supp}(f)$ in $(b-\delta(b), b)$. It follows that $O=F^{+} \cap \operatorname{supp}(f) \cap(b-\delta(b), b)$ is not empty.

Now $F^{+}$is $G_{\delta^{-}}$set, and consequently so is $O$. By the Baire Category Theorem there is an integer $n$ and an interval $K \subset(b-\delta(b), b)$ such that $K \cap O \neq \varnothing$ and $\{z \mid z \in O \cap K$ and $\delta(z)>1 / n\}$ is dense in $K \cap O$. Without loss of generality we may suppose that the length of $K$ is less than $1 / n$.

The function $f^{+}$is not integrable on $K$, and so there is a $\delta$-fine Perron partition $P$ of $K$ such that $\sigma\left(f^{+}, P\right)>10$. We may adjust this $P$ so that every point is in the interior of its corresponding interval, perhaps slightly shrinking $K$ in the meantime. Now suppose that $I_{i}$ is an interval of $P$ whose corresponding point $x_{i}$ has $f\left(x_{i}\right)<0$. Since $f^{+}$is not integrable on any neighborhood of $x_{i}$, there is a point $y_{i}$ in the interior of the interval $I_{i}$ with $f\left(y_{i}\right)>0$. Then there is a point $z_{i}$ of $F^{+}$in the interior of $I_{i}$ with $\delta\left(z_{i}\right)>1 / n$. In the partition $P$ replace the point $x_{i}$ by $z_{i}$. Do this for every point of the partition $P$ at which $f$ has a negative value. We obtain a new partition $P^{\prime}$ which is $\delta$-fine and for which $\sigma\left(f, P^{\prime}\right)=\sigma\left(f^{+}, P^{\prime}\right) \geqslant \sigma\left(f^{+}, P\right)>10$, contradicting the choice of $\delta$.

This completes the proof of our result. Since $f$ is certainly Lebesgue integrable on a neighborhood of each point not in $\operatorname{supp}(f)$, we have the following.

THEOREM. If $f$ is $\alpha$-measurable and has locally small Riemann sums with respect to $\alpha$ on the interval $I$, then $\{x \mid f$ is Lebesgue integrable on a neighborhood of $x\}$ is dense in $I$.

\section{Structure of the set on which $f$ is not Perron integrable}

Our intention of course is to prove that this is empty. To do this we must build up a store of information regarding its structure. No supposition of $\alpha$-measurability is required for the results of this section. 
THEOREM. If the function $f$ has locally small Riemann sums with respect to $\alpha$ on the interval $[a, b]$ and if $f$ is $P$-integrable with respect to $\alpha$ on each open subinterval of $[a, b]$, then if is $P$-integrable with respect to $\alpha$ on $[a, b]$.

Proof. We show that $f$ is $P$-integrable "at $b$ ", the result for the other endpoint being exactly similar. If $\alpha$ is not continuous at $b$, then set

$$
\alpha^{\prime}(x)= \begin{cases}\alpha(x) & \text { if } x<b, \\ \lim _{t \rightarrow b^{-}} \alpha(t) & \text { if } x=b,\end{cases}
$$

and set $\alpha^{\prime \prime}(x)=\alpha(x)-\alpha^{\prime}(x)$. Then $\alpha=\alpha^{\prime}+\alpha^{\prime \prime}$, and certainly $f$ satisfies the hypotheses of the theorem with respect to $\alpha^{\prime}$. Since $f$ is integrable with respect to $\alpha^{\prime \prime}$, we will be done if it is integrable with respect to $\alpha^{\prime}$, which is continuous at $b$.

With respect to the function $\alpha^{\prime}$ there are no improper Perron integrals [3, page 37], so we need only show that $\lim _{t \rightarrow b^{-}} \int_{a}^{1} f d \alpha^{\prime}$ exists. To do this we need only show that for each positive $\varepsilon$ there is a positive $r$ such that $\left|\int_{a}^{t} f d \alpha^{\prime}\right|<\varepsilon$ whenever $b-r<s<t<b$.

Since $f$ has locally small Riemann sums at $b$, there is a positive function $\delta$ such that $\left|\sigma\left(f, P, \alpha^{\prime}\right)\right|=|\sigma(f, P, \alpha)|<\varepsilon$ whenever $P$ is a $\delta$-fine Perron partition of an interval in $(b-\delta(b), b)$. If $b-\delta(b)<s<t<b$, then any $\delta$-fine Perron partition of $[s, t]$ must satisfy $|\sigma(f, P)|<\varepsilon$, and since $f$ is $P$-integrable on $[s, t]$, we must have $\left|\int_{s}^{t} f d \alpha^{\prime}\right| \leqslant \varepsilon$. This establishes the theorem.

COROLLARY. If the function $f$ has locally small Riemann sums with respect to $\alpha$ on the interval $I$, then the set $C=\{x \mid f$ is not P-integrable with respect to $\alpha$ on a neighborhood of $x\}$ has no isolated points.

\section{Proof of the main theorem}

Suppose we are given in $\alpha$-measurable function $f$ which has locally small Riemann sums with respect to $\alpha$ on the interval $I$. Suppose that the set $C$ in the immediately preceding corollary is not empty. The results of Section 3 imply that $C$ is nowhere dense in $I$, and the corollary says that $C$ has no isolated points. Since $C$ is certainly closed in $I, C$ must be a nowhere dense perfect set. Let $O=I-C$. Let the function $f_{C}$ agree with $f$ on $C$ and be equal to 0 elsewhere and let $f_{O}=f-f_{C}$.

Let $U=(a, b)$ be any component of $O$. From Section 4 we know that $f$ is $P$-integrable on $[a, b]$, and so we can define the (finite) number $M(U)=$ $\sup \left\{\left|\int_{x}^{y} f d \alpha\right| \mid a \leqslant x<y \leqslant b\right\}$. 
Suppose that $f_{C}$ is $P$-integrable on some interval $K$ which contains a point of $C$ in its interior. If $\Sigma\{M(U) \mid U \subset W\}$ converges for some interval $W$ which is contained in $K$ and has a point of $C$ in its interior, then it is not hard to show that $f_{O}$ is $P$-integrable on $W$, which is a contradiction. (This is essentially the Harnack property of the Perron integral.)

Let $x$ belong to $C \cap K^{\circ}$. There is a positive function $\delta$ such that $\left|\sigma\left(f_{O}, P\right)\right|<1$ whenever $P$ is a Perron partition of an interval in $(x-\delta(x), x+\delta(x))-\{x\}$. Using the Baire Category Theorem, we can produce an integer $n$ and an interval $J$ of length less than $1 / n$ such that $J$ lies in $(x-\delta(x), x+\delta(x))-\{x\}$, $J \cap C \neq \varnothing, D=\{x \mid x \in C$ and $\delta(x)>1 / n\} \cap J$ is dense in $J \cap C$ and $J \subset$ $K^{\circ}$.

Now $\Sigma\{M(U) \mid U$ is a component of $O$ lying in $J\}$ does not converge, so without loss of generality there are pairwise disjoint intervals $K_{1}, K_{2}, \ldots, K_{q}$ which lie in distinct components of $O$ lying in $J$, and for which $\sum_{i=1}^{q} \int_{K_{i}} f d \alpha>10$. Each such $K_{i}$ has a $\delta$-fine Perron partition $P_{i}$ such that $\sum_{i=1}^{q} \sigma\left(f, P_{i}\right)>10$.

Using the facts that (1) between any two $K_{i}$ 's there must be points of $C$, (2) $D$ is dense in $J \cap C$ and (3) the length of $J$ is less than $1 / n$, it is easy to use points of $D$ to complete the partitions $P_{1}, P_{2}, \ldots, P_{q}$ to a partition $P$ of $J$. Since $f=f_{O}$ on $O$, we have $\sigma\left(f, P_{i}\right)=\sigma\left(f_{0}, P_{i}\right)$ for each $i$. Since $f_{O}(x)=0$ for $x$ in $D$, we have $\sigma\left(f_{O}, P\right)>10$, contradicting the property of $\delta$.

Since the above argument applies in any neighborhood of any point of $C$, we conclude that $f_{C}$ cannot be $P$-integrable on any neighborhood of any point of $C$.

Suppose the function $f_{C}^{-}$is integrable on an interval $W$ which includes a point $x$ of $C$ in its interior. Let $\delta$ be the positive function delivered by the locally small Riemann sum property for this $x$, the function $f$ and the bound 1 on Riemann sums. Let $L$ be an interval contained in $W$ and in $(x-\delta(x), x+\delta(x))-\{x\}$, and which contains a point of $C$ in its interior. Then $f_{C}^{-}$is integrable on $L$, and consequently $f_{C}^{+}$is not. Since we are dealing with measurable functions, we can assume that $\{x \mid x \in L \cap C$ and $f(x) \geqslant 0\}$ is a $G_{\delta}$-set. Therefore we can apply the Baire Category Theorem to produce an integer $n$ and an interval $K$ of length less than $1 / n$ which lies in $L$ and for which $\{x \mid x \in K \cap C, f(x) \geqslant 0$ and $\delta(x)>$ $1 / n\}$ is dense in the non-empty set $\{x \mid x \in K \cap C$ and $f(x) \geqslant 0\}$.

Let $\delta^{\prime}$ be a positive function such that (1) $\delta^{\prime}<\delta$, (2) $\delta^{\prime}(x)<d(x, C)$ when $x \notin C$, (3) if a component $U$ of $O$ meets $\left(z-\delta^{\prime}(z), z+\delta^{\prime}(z)\right)$, then the closure of $U$ lies in $(z-\delta(z), z+\delta(z))$ and (4) $\sigma\left(f_{C}^{-}, P\right)$ is within 1 of $\int_{K} f_{C}^{-} d \alpha$ whenever $P$ is a $\delta^{\prime}$-fine partition of $K$. Since $f_{C}^{+}$is not integrable on $K$, there is a $\delta^{\prime}$-fine Perron partition $P$ of $K$ such that $\sigma\left(f_{C}^{+}, P\right)>\int_{K} f_{C}^{-} d \alpha+10$.

Suppose some point of $P$ belongs to $O$. Then by property (2) of $\delta^{\prime}$ the interval corresponding to this point lies in a component of $O$. An endpoint of this component belongs to another interval of $P$, and by property (3) of $\delta^{\prime}$ this 
interval can be extended so as to contain the entire component except for the portion already contained in the interval of $P$ containing the other endpoint of the component. By doing this for each point of $P$ belonging to $O$ we construct a new $\delta$-fine Perron partition $P$, and in so doing we do not decrease the sum $\sigma\left(f_{C}^{+}, P\right)$. Further, each point of this new partition $P$ belongs to $C$.

Now because the points of $P$ all belong to $C$, we have $\sigma(f, P)=\sigma\left(f_{C}^{+}, P\right)-$ $\sigma\left(f_{C}^{-}, P\right)$. The latter sum is within 1 of $\int_{K} f_{C}^{-} d \alpha$, and the former is larger than this integral plus 10. Consequently, the size of $\sigma(f, P)$ contradicts the property of the function $\delta$.

The same argument will apply when $f_{C}^{+}$is integrable on a neighborhood of some point of $C$, so we must conclude that neither $f_{C}^{-}$nor $f_{C}^{+}$are integrable on any neighborhood of any point of $C$.

Now let $x$ be any point of $C$ and let $\delta$ be the positive function furnished by the locally small Riemann sum property for this $x$ and the bound 1 for the Riemann sums. As before an application of the Baire Category Theorem yields an integer $n$ and an interval $K$ of length less than $1 / n$ which lies within $\delta(x)$ of $x$ but does not include $x$, and for which the set $D=\{z \mid z \in K \cap C, f(z) \geqslant 0$ and $\delta(z)>$ $1 / n\}$ is dense in the nonempty set $\{z \mid z \in K \cap C$ and $f(z) \geqslant 0\}$. Since $f_{C}^{+}$is not integrable on $K$, there is a $\delta$-fine Perron partition $P$ of $K$ for which $\sigma\left(f_{C}^{+}, P\right)>10$. By using a function $\delta^{\prime}$ as we did above, we can further require that every point of $P$ belongs to $C$ and that every point of $P$ is in the interior of its corresponding interval. Since $f_{C}^{+}$is not integrable on any neighborhood of any point of $C$, it follows that near any point $z$ of $P$ for which $f(z)<0$ there are many points $x$ of $C$ for which $f(x)>0$. The denseness of the set $D$ then furnishes such a point $x$ by which $z$ may be replaced in the partition. These replacements then deliver a $\delta$-fine partition $P^{\prime}$ for which $\sigma\left(f, P^{\prime}\right)=\sigma\left(f_{C}^{+}, P^{\prime}\right)>10$. This contradicts the property of $\boldsymbol{\delta}$.

We have arrived at our final contradiction. Each contradiction in this long trail arose because we assumed that the set $C$ of points which have no neighborhood on which $f$ is $P$-integrable is not empty. Consequently that set must be empty, and our main theorem is proved.

\section{Lebesgue and Riemann integrals}

Some comments are in order regarding the applicability of the locally small Riemann sum criterion to Lebesgue and Riemann integrals.

Let us first consider Riemann integration. The generalized Riemann definition of Perron integrability clearly becomes exactly the definition of Riemann integrability when we add the requirement that the function $\delta$ be constant. However, 
even with this requirement in the definition of locally small Riemann sums, every bounded measurable function has locally small Riemann sums. Consequently, this paper's criterion for integrability does not specialize very well, if at all, to the Riemann case.

One could hope, however, that the criterion would work well for the Lebesgue case by requiring in its definition that the Riemann sums are small for any partition of the small intervals, not just for Perron partitions. This is not the case, and we outline an example.

Let the function $f$ be the derivative of the function whose value at $x$ is 0 when $x$ is 0 and $x^{2} \sin \left(1 / x^{2}\right)$ when $x$ is not 0 . It is well-known that $f$ is not Lebesgue integrable on $[0,1]$ but that it is Perron integrable there. (We use $\alpha(x)=x$ throughout this section.)

That $f$ has locally small Riemann sums for general partitions at points of $(0,1]$ is clear, since $f$ is even continuous there. So we need worry only about the point 0 . Let $\varepsilon$ be positive. There is a positive function $\delta_{n}$ on the interval $J_{n}=$ $[1 /(n+1), 1 / n]$ such that, for any two $\delta_{n}$-fine partitions $P$ and $Q$ of $J_{n}$, we have $|\sigma(f, P)-\sigma(f, Q)|<\varepsilon / 2^{n}$. There is also a positive function $\delta$ such that $|\sigma(f, P)|$ $<\varepsilon / 2$ whenever $P$ is a $\delta$-fine Perron partition of an interval in $(0, \delta(0))$. We can then find a positive function $\delta^{\prime}$ smaller than $\delta$ and so small that any partition of an interval in $\left(0, \delta^{\prime}(0)\right)$ breaks up into partitions of intervals in the $J_{n}$ which are $\delta_{n}$-fine. Putting all these requirements together shows that $|\sigma(f, P)|<\varepsilon$ whenever $P$ is any $\delta^{\prime}$-fine partition of an interval in $\left(0, \delta^{\prime}(0)\right)$, so that $f$ has locally small Riemann sums for general partitions even though $f$ is not Lebesgue integrable.

However, there is a formulation of the locally small Riemann sum property that is valid for Lebesgue as well as Perron integration. First, set

$$
\alpha^{\prime}(b)=\lim _{t \rightarrow 0} \alpha([b-t, b+t]) .
$$

We say that a function $f$ on the interval $I$ has Property LSRS on $I$ with respect to $\alpha$ if, for each $b$ in $I$, and for each positive number $\varepsilon$, there is a positive function $\delta$ on $I$ for which $\left|\sigma(f, P)-f(b) \alpha^{\prime}(b)\right|<\varepsilon$ whenever $P$ is a $\delta$-fine partition of an interval containing $b$ in its interior and lying in $(b-\delta(b), b+$ $\delta(b))$. Property LSRS-P is the same except that the condition need hold only for Perron partitions $P$. Then Property LSRS-P is equivalent to the property of having locally small Riemann sums as defined in this paper. Further, one can show that an $\alpha$-measurable function $f$ on the interval $I$ is Lebesgue-Stieltjes integrable with respect to $\alpha$ on $I$ if and only if $f$ has Property LSRS with respect to $\alpha$ on $I$. Details of these results will appear in the Proceedings of the American Mathematical Society. 


\section{References}

[1] R. M. McLeod, The generalized Riemann integral (Carus Mathematical Monograph No. 20, American Mathematical Association, Washington, D. C., 1980).

[2] I. P. Natanson, Theory of functions of a real variable, Vol. II (translated by L. F. Boron, Frederick Ungar Publishing Co., New York, 1960).

[3] W. F. Pfeffer, 'The Riemann-Stieltjes approach to integration' (Technical Report 187, National Research Institute for Mathematical Sciences, Pretoria, South Africa, 1980).

[4] S. Saks, Theory of the integral (2nd revised edition, translated by L. C. Young, Dover Publications, New York, 1964).

[5] A. Schurle, 'Perron integrability versus Lebesgue integrability', Canad. Math. Bull. 28 (1985), 463-468.

Department of Mathematical Sciences

University of Petroleum and Minerals

UPM Box 639

Dhahran 31261

Saudi Arabia 